\title{
Predicting observational signatures of coronal heating by Alfvén waves and nanoflares
}

\author{
Patrick Antolin ${ }^{1}$, Kazunari Shibata ${ }^{1}$, Takahiro Kudoh ${ }^{2}$, Daiko \\ Shiota $^{2}$, and David Brooks ${ }^{3} \dagger$ \\ ${ }^{1}$ Kwasan Observatory, Kyoto University, \\ Yamashina, Kyoto, 607-8471, Japan \\ email: antolin@kwasan.kyoto-u.ac.jp, shibata@kwasan.kyoto-u.ac.jp \\ ${ }^{2}$ National Astronomical Observatory of Japan, \\ 2-21-1, Osawa, Mitaka, Tokyo, 181-8588, Japan \\ email: kudoh@th.nao.ac.jp, shiota@cfca.jp \\ ${ }^{3}$ Space Science Division, Naval Research Laboratory, \\ Washington, DC 20375, USA \\ email: dhbrooks@ssd5.nrl.navy.mil
}

\begin{abstract}
Alfvén waves can dissipate their energy by means of nonlinear mechanisms, and constitute good candidates to heat and maintain the solar corona to the observed few million degrees. Another appealing candidate is the nanoflare-reconnection heating, in which energy is released through many small magnetic reconnection events. Distinguishing the observational features of each mechanism is an extremely difficult task. On the other hand, observations have shown that energy release processes in the corona follow a power law distribution in frequency whose index may tell us whether small heating events contribute substantially to the heating or not. In this work we show a link between the power law index and the operating heating mechanism in a loop. We set up two coronal loop models: in the first model Alfvén waves created by footpoint shuffling nonlinearly convert to longitudinal modes which dissipate their energy through shocks; in the second model numerous heating events with nanoflare-like energies are input randomly along the loop, either distributed uniformly or concentrated at the footpoints. Both models are based on a 1.5-D MHD code. The obtained coronae differ in many aspects, for instance, in the simulated intensity profile that Hinode/XRT would observe. The intensity histograms display power law distributions whose indexes differ considerably. This number is found to be related to the distribution of the shocks along the loop. We thus test the observational signatures of the power law index as a diagnostic tool for the above heating mechanisms and the influence of the location of nanoflares.
\end{abstract}

Keywords. Sun: corona, Sun: flares, (magnetohydrodynamics:) MHD, waves

\section{Introduction}

The coronal heating problem, the heating of the solar corona up to a few hundred times the average temperature of the underlying photosphere, is one of the most perplexing and to date unresolved problems in astrophysics. Alfvén waves produced by the constant turbulent convective motions in the sub-photospheric region (Alfvén 1947) have been shown to transport enough energy to heat and maintain a corona (Uchida \& Kaburaki 1974; Wentzel 1974). This is known as the the Alfvén wave heating model (Hollweg, Jackson \& Galloway 1982; Kudoh \& Shibata 1999). Many dissipating mechanisms for Alfvén waves have been proposed, such as mode conversion, phase mixing or resonant absorption

$\dagger$ Also at George Mason University, 4400 University Drive, Fairfax, VA 22020, USA. 
(see reviews by e.g. Erdélyi 2004; Erdélyi \& Ballai 2007 and further references therein). Another promising coronal heating candidate mechanism is the nanoflare-reconnection heating model. The nanoflare-reconnection process was first suggested by Parker (1988), who considered a magnetic flux tube as being composed by a myriad of magnetic field lines braided into each other by continuous footpoint shuffling. Many current sheets in the magnetic flux tube would be created that would lead to many magnetic reconnection events, releasing energy impulsively and sporadically in small quantities of the order of $10^{24} \mathrm{erg}$ or less (nanoflares). The energy flux carried by the slow modes generated in the reconnection events is expected to be one order of magnitude higher than the energy flux carried by the generated Alfvén waves (Takeuchi \& Shibata 2001). Hence, in this picture, the corona would be heated mainly by the accumulation of numerous nanoflares coming from reconnection events and by magnetoacoustic shocks.

Observations with instruments such as TRACE (Krucker \& Benz 1998; Parnell \& Jupp 2000) and Yohkoh/SXT (Katsukawa \& Tsuneta 2001) have shown that nanoflares in the corona are rather impulsive and ubiquitous in character thus supporting the nanoflarereconnection scenario. The intermittent behaviour of coronal loops, and their modelling by random energy deposition representing nanoflares of locally damped wave heating was studied by Mendoza-Briceño et al. (2002, 2005) and Mendoza-Briceño \& Erdélyi (2006). However, Moriyasu et al. (2004), showed that the observed spiky intensity profiles due to impulsive releases of energy could also be specifically obtained from nonlinear Alfvén wave heating. The resulting shock heating was found to be episodic, impulsive and uniformly distributed throughout the corona implying that the observed nanoflares may be directly related to Alfvén waves rather than to magnetic reconnection.

It has been shown that energy release processes in the Sun, from solar flares down to microflares, follow a power law distribution in frequency with an index (slope) around -1.6 (Shimizu 1995). Hudson (1991) showed that if smaller energetic events such as nanoflares have a power law distribution with an index steeper than -2 then they would represent the bulk of the heating in the corona. However, measurements of this quantity have shown a large range of values (cf. table 1 in Benz \& Krucker 2002; Aschwanden 2004).

In Taroyan et al. (2007) it is shown that an analysis of power spectra of Doppler shift time series allows to differentiate between uniformly heated loops from loops heated near their footpoints. Taking into account the uniform heating nature resulting from Alfvén waves this idea could also allow to differentiate Alfvén wave heated loops from loops heated by mechanisms concentrating towards the footpoints. Following this idea, in this work we propose a way to discern observationally between Alfvén wave heating and nanoflare-reconnection heating. The proposed idea constitutes also a diagnostic tool for the location of the heating along coronal loops. It relies on the fact that the distribution of the shocks in loops differs substantially between the two models, due to the different characteristics of the wave modes they produce. As a consequence, X-ray intensity profiles differ substantially between an Alfvén wave heated corona and a nanoflare heated corona. The frequency distribution of the heating events obtained from the intensity profiles is found to follow a power law distribution in both cases, with indexes (slopes) which differ significantly from one heating model to the other, depending also on the 'observed' region of the magnetic flux tube. We thus analyze the link between the power law index of the frequency distribution and the operating heating mechanism in the loop. 


\section{Models}

\subsection{Geometry, initial conditions and numerical code}

The model is essentially the same as in Moriyasu et al. (2004). It consists of a magnetic flux tube of $100 \mathrm{Mm}$ in length whose geometry takes into account the observed expansion of magnetic flux in the photosphere and chromosphere, with an apex-to-base area ratio of 1000 (cf. Fig. 1 in Moriyasu et al. 2004). A single external poloidal magnetic field line is considered ( $s$ component) along which azimuthal rigid motions ( $\phi$ components of velocity and magnetic fields) are allowed (1.5-dimensional approximation). Conservation of magnetic flux implies $B_{s}=B_{0}\left(r_{0} / r\right)^{2}$, where $B_{0}$ is the value of the magnetic field at the photosphere and $r_{0}=200 \mathrm{~km}$ is the initial radius of the loop. In the photosphere the value of $\beta=8 \pi p / B_{s}^{2}$ (the ratio of gas to magnetic pressures) is unity. We assume an inviscid perfectly conducting fully ionized plasma. The effects of thermal conduction and radiative cooling are considered. The Spitzer conductivity corresponding to a fully ionized plasma is considered. An optically thin approximation formula for radiative losses is adopted (cf. Table 1 in Hori et al. 1997), but we assume that the plasma becomes optically thick for temperatures below $4 \times 10^{4} \mathrm{~K}$. In this case, radiative losses $R$ are approximated by $R(\rho)=4.9 \times 10^{9} \rho$ (Anderson \& Athay 1989). The initial temperature of the loop is set at $T=10^{4} \mathrm{~K}$ everywhere. The loop is assumed to follow hydrostatic pressure balance from the photosphere to a height of $800 \mathrm{~km}$. For the rest of the loop, density decreases as $\rho \propto h^{-4}$, where $h$ is the height from the base of the loop (Shibata et al. 1989a, 1989b). The photospheric density and pressure are respectively $\rho_{0}=2.53 \times 10^{-7} \mathrm{~g} \mathrm{~cm}^{-3}$ and $p_{0}=2.09 \times 10^{5} \mathrm{dyn} \mathrm{cm}^{-2}$. The value for the magnetic field at the photosphere is $B_{0}=2.29 \times 10^{3} \mathrm{G}$ and decreases to $B_{\text {apex }}=2.29 \mathrm{G}$ at the apex of the loop.

The spatial resolution is $5 \mathrm{~km}$ up to a height of $16 \mathrm{Mm}$, after which it is allowed to increase gradually to $20 \mathrm{~km}$ (which is the adopted resolution in Moriyasu et al. 2004 for the entire loop). We take rigid wall boundary conditions at the photosphere. The numerical schemes adopted are the CIP scheme (Yabe \& Aoki 1991) and the MOCCT scheme (Evans \& Hawley 1988; Stone \& Norman 1992). Please refer to Kudoh, Matsumoto \& Shibata (1998) for details about the application of these schemes.

\subsection{Heating models}

In the Alfvén wave heating model, Alfvén waves are generated by a photospheric driver represented by a torque added to the $\phi$-component of the momentum equation. The amplitude of the torque is random, hence creating a white noise Alfvén wave spectrum. This model is essentially equivalent to a $1.5-\mathrm{D}$ model with radial perturbations, except for a centrifugal force which is of small importance compared to the other forces. The potential of 1.5-D MHD models of torsional Alfvén waves has already been considered in the past for the formation of spicules (e.g. Kudoh \& Shibata 1999, James \& Erdélyi 2002) and coronal heating (e.g. Moriyasu et al. 2004).

The nanoflare model is basically one dimensional and is similar to the model of Taroyan et al. 2006. Heating events simulating nanoflares are input randomly throughout the loop as artificial perturbations in the internal energy of the gas, generating only magnetoacoustic modes. This approach is justified by 2-D simulations of photospheric reconnection by Takeuchi \& Shibata (2001) in which it is shown that the energy flux carried by Alfvén waves is negligible with respect to the flux carried by the slow magnetoacoustic waves. The spatial distribution of the heating in coronal loops is a controversial point. Observational evidence for footpoint heating (Aschwanden et al. 2001), uniform heating (Priest et al. 1998) or apex heating (Reale 2002) has been found. Here we consider nanoflares distributed towards the footpoints ('footpoint heating'; see Mendoza-Briceño et al. 2002) 
Table 1. Mean input values for the nanoflare model

\begin{tabular}{ccccc}
\hline $\begin{array}{c}\text { Event } \\
\text { distribution }\end{array}$ & $\begin{array}{c}\text { Energy } \\
\text { distribution }\end{array}$ & $\begin{array}{c}\text { Number } \\
\text { of runs }\end{array}$ & $\begin{array}{c}\langle\text { Energy } \\
\min -\max \end{array}$ & $\begin{array}{c}\langle\text { Flux }\rangle\left(\mathrm{erg} \mathrm{cm}{ }^{-2} \mathrm{~s}^{-1}\right) \\
\min -\max \end{array}$ \\
\hline $\begin{array}{c}\text { loop } \\
\text { footpoint }\end{array}$ & uniform & 4 & $8.2 \times 10^{24}-8.2 \times 10^{26}$ & $2.5 \times 10^{5}-2.5 \times 10^{7}$ \\
uniform & 10 & $1.3 \times 10^{23}-4.7 \times 10^{26}$ & $4.7 \times 10^{5}-2.5 \times 10^{7}$ \\
foop & power law & 4 & $9.3 \times 10^{24}-2.9 \times 10^{25}$ & $2.1 \times 10^{6}-6.6 \times 10^{6}$ \\
power law & 10 & $4.9 \times 10^{24}-2.2 \times 10^{25}$ & $4.8 \times 10^{6}-2.1 \times 10^{7}$ \\
\hline
\end{tabular}

The first column denotes the spatial distribution of the heating, either uniformly distributed along the loop ('loop') or concentrated at the footpoints ('footpoint'). The energy distribution among the events can be uniform or can follow a power law with a certain index, corresponding, respectively, to 'uniform' and 'power law' in the second column. The third column denotes the number of runs performed with such heating distribution. In the fourth and fifth columns we have, respectively, the extremum values among the runs for the mean energy per event and for the mean energy flux (minimum and maximum).

as well as uniformly (randomly) distributed along the loop ('uniform heating'). We adopt the same form of the heating function for each event as in Taroyan et al. 2006. Additionally, the volumetric energies $E_{0}$ of the events can be distributed either uniformly or as a power law as reported by observations (Shimizu 1995). The probability density function of the volumetric heating $E_{0}$ in the later case is then of the form $d N\left(E_{0}\right) / d E_{0} \propto E_{0}^{\alpha}$ where $N\left(E_{0}\right)$ is the number of heating events having a volumetric heating between $E_{0}$ and $E_{0}+d E_{0}$ and $\alpha$ is the power law index of the distribution.

The time scale of the heating in a reconnection event depends on the dimension of the current sheet which is very hard to estimate. If the length across the reconnection region is of the order of $\sim 1000 \mathrm{~km}$, the time scale of a (small) reconnection event leading to a nanoflare should oscillate between 1 and $10 \mathrm{~s}$, since the order of the Alfvén speed in the chromosphere and in the corona is, respectively, $\sim 100 \mathrm{~km} \mathrm{~s}^{-1}$ and $\sim 1000 \mathrm{~km} \mathrm{~s}^{-1}$. This value however is not established. Here we consider runs with maximum duration times for a heating event of $10 \mathrm{~s}$ or $40 \mathrm{~s}$. Different values per run of the heating scale length are also tried: 200, 500 or $1000 \mathrm{~km}$. The frequency of the heating events can be either $1 / 50,1 / 34$ or $1 / 7 \mathrm{~s}^{-1}$. For footpoint concentrated heating the events are randomly distributed in the loop segments [2,20] $\mathrm{Mm}$ and [70,98] $\mathrm{Mm}$, or [2,12] $\mathrm{Mm}$ and $[88,98]$ $\mathrm{Mm}$, or $[1,10] \mathrm{Mm}$ and $[90,99] \mathrm{Mm}$, where length is measured from one footpoint. For uniform heating along the loop the events can occur in the range [2,98] $\mathrm{Mm}$. For runs with uniformly distributed energies we take $E_{0}=0.01,0.05$ or $0.5 \mathrm{erg} \mathrm{cm}^{-3} \mathrm{~s}^{-1}$. For runs with energies that follow a power law distribution in frequencies we take as minimum and maximum volumetric energies $0.005 \mathrm{erg} \mathrm{cm}^{-3} \mathrm{~s}^{-1}$ and $10 \mathrm{erg} \mathrm{cm}^{-3} \mathrm{~s}^{-1}$ respectively. Several values for the power law index $\alpha$ are considered, ranging from -1.5 to -2.2 . In Table 1 we calculate the extremum values among the runs for the mean energy per event and for the mean energy flux.

\section{Numerical results}

\subsection{From nonlinear Alfvén waves}

The left column panels of Fig. 1 show the time profiles at times $t=0 \mathrm{~min}$ (dotted line), $t=33.5 \mathrm{~min}$ (dashed line) and $t=270.7 \mathrm{~min}$ (solid line) of the temperature, density, poloidal velocity and the ratio of the toroidal (azimuthal) and poloidal components of the magnetic field for a typical case of a loop heated by Alvén waves. In this case, waves are generated by a photospheric velocity field satisfying $\left\langle v_{\phi}^{2}\right\rangle^{1 / 2}=1.9 \mathrm{~km} \mathrm{~s}^{-1}$, where the average is taken over the entire simulation time. As shown in Kudoh \& Shibata (1999) and in Moriyasu et al. (2004), due to nonlinear effects Alfvén waves convert to longitudinal slow and fast modes when propagating from the photosphere to the 
chromosphere which then steepen into slow and fast shocks, respectively. Mode conversion and the subsequent shock formation also happens everywhere in the corona. This can be understood from the bottom panel of Fig. 1 which shows the ratio $B_{\phi} / B_{s}$ of the toroidal (azimuthal) component to the poloidal component of the magnetic field. This quantity becomes high episodically, not only in the chromosphere but also in the corona. When this happens nonlinear effects are large and energy is transfered from the Alfvén mode to the longitudinal slow and fast modes, which steepen into shocks and heat the plasma. The heating from the shocks is episodic and uniformly distributed throughout the corona. The loop gradually heats from chromospheric to coronal temperatures, the coronal part of the loop increasing in length and becoming flatter with time due to the uniformly distributed shock heating and to thermal conduction. After $t=150 \mathrm{~min}$ approximately, the loop reaches a quasi-steady state in which radiative losses, thermal conduction and shock heating are in balance. As shown in Moriyasu et al. (2004), Alfvén wave heated loops satisfy the RTV scaling law (Rosner, Tucker, Vaiana 1974). Although in steady state, the emerging corona is characterized by a highly dynamical state, with flow velocities in the corona reaching $200 \mathrm{~km} \mathrm{~s}^{-1}$ at various times (Fig. 1). The transition region is highly dynamical as well. The potential of the Alfvén wave model for creating spicules and other chromospheric dynamical events was already shown by Kudoh \& Shibata 1999.

\subsection{From nanoflares}

In the center and right column panels of Fig. 1 the time profiles at $t=0 \mathrm{~min}, t=33.5 \mathrm{~min}$ and $t=270.7 \mathrm{~min}$ of the temperature, density and poloidal velocity along the loop are shown for two typical cases of nanoflare heating. The center column panels correspond to a loop whose heating events are concentrated at the footpoints (in the loop range $[1,10]$ $\mathrm{Mm}$ and $[90,99] \mathrm{Mm})$. For this run, the mean energy per event is $6.6 \times 10^{24} \mathrm{erg}$ and the mean energy flux is $6.4 \times 10^{6} \mathrm{erg} \mathrm{cm}^{-2} \mathrm{~s}^{-1}$. The right column panels correspond to a loop which heating events are uniformly distributed along the loop (in the loop range [2,98] $\mathrm{Mm})$. The mean energy per event is $4.09 \times 10^{25} \mathrm{erg}$ and the mean energy flux is $1.27 \times 10^{6}$ erg $\mathrm{cm}^{-2} \mathrm{~s}^{-1}$. In both cases the energy flux deposited by the heating events is enough to heat the loop to coronal values, agreeing with the results by Mendoza-Briceño et al. 2002. The heating events create sporadic bumps in the temperature profile which are however rapidly flattened by thermal conduction. As already pointed out by MendozaBriceño, Sigalotti \& Erdélyi (2005), the local bumpy profiles close to the transition region may resemble the intermittent behavior reported by Patsourakos \& Vial (2002) from an analysis of light-curves from transition region and low coronal lines, simultaneously recorded in a quiet Sun region by $S o H O /$ SUMER. A heating event produces a local increase in pressure which propagates as an acoustic wave. Fig. 1 shows that shocks produced by heating events in the nanoflare model are weaker than shocks from the slow and fast modes produced by Alfvén waves. For footpoint concentrated heating we have strong shocks basically only near the location of the heating events, close to the transition region. By the time the strong shocks reach the apex they have dissipated most of their energy. Heating events occur frequently above the transition region pushing it downwards. Due to the high frequency with which they occur (one to ten events per minute) the loop reaches a thermal equilibrium in which the transition region is at a relatively low height $(\sim 5000 \mathrm{~km}$ for the loop in Fig. 1, center column) with no spicule formation. For uniformly distributed heating events the damping effect is more dramatic: shocks are weak everywhere. This is due to the higher coronal temperatures achieved in the uniform heating case, which make flattening of shocks from thermal conduction much more effective. The large difference of the nanoflare heating model with the Alfvén 


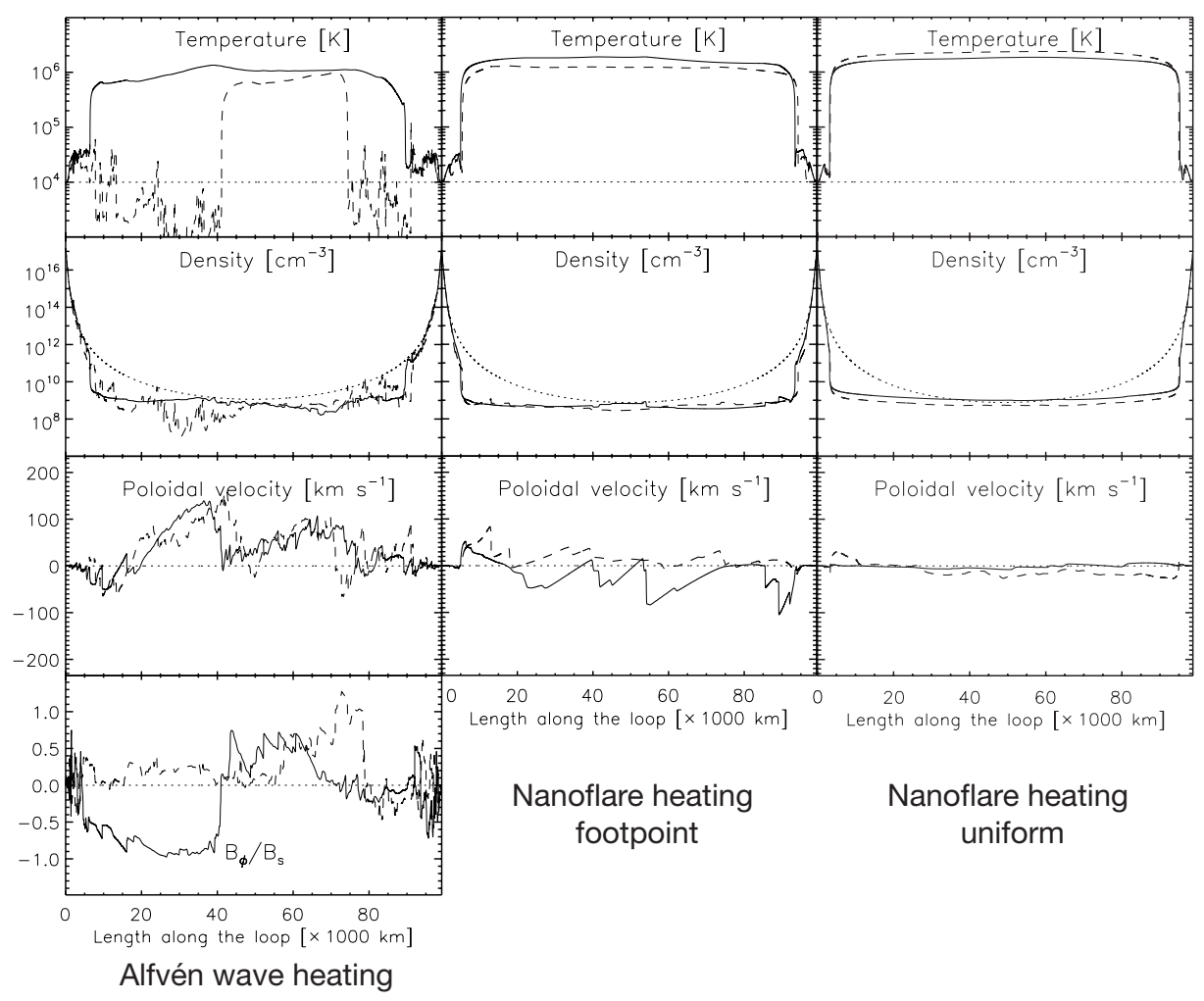

Figure 1. Profiles of quantities along the loop at various times for a loop heated by Alfvén waves (left column), a loop with heating events simulating nanoflares concentrated at the footpoints (center column) or uniformly distributed along its length (right column). In the case of Alfvén wave heating, the Alfvén waves are generated by a photospheric velocity field of $\left\langle v_{\phi}^{2}\right\rangle^{1 / 2}=1.9$ $\mathrm{km} \mathrm{s}^{-1}$. Rows indicate, from top to bottom respectively, profiles along the loop for temperature, density and poloidal velocity. The bottom panel of the left column shows the toroidal to poloidal magnetic field ratio for the case where Alvén waves are present. The profiles are plotted for $t=0$ $\min$ (dotted line), $t=33.5 \mathrm{~min}$ (dashed line) and $t=270.7 \mathrm{~min}$ (solid line).

wave heating model is basically due to the combination of two effects: the nonlinear conversion from Alfvén waves to longitudinal slow and fast modes occurs everywhere along the corona, resulting in ubiquitous strong shocks. Also, fast shocks resulting from this conversion dissipate with much less efficiency than slow shocks, which are the only shocks found in the nanoflare model. Hence, strong fast shocks will remain strong shocks for larger times, making a more dynamical corona.

\section{Predicting observable features}

We now express the results in terms of observable quantities. We construct the intensity flux profiles and intensity histograms with the help of Hinode/XRT response function (using the thin $\mathrm{Al}$ mesh filter). Fig. 2 shows time series of the intensity flux calculated at the top of the transition region (top panels) and at the apex of the loop (bottom panels). From left to right the columns show, respectively, a loop heated by Alfvén waves, a loop with heating events concentrated at the footpoints with a power law energy spectrum with an index of $\alpha=-1.8$, and a loop with heating events uniformly distributed along the loop and whose energies are uniformly distributed in frequency. In Fig. 3 we show 
intensity histograms constructed from the intensity fluxes of Fig. 2. The panels of Fig. 3 are distributed in the same way as the panels of Fig. 2. We can see that all panels display distributions which can be approximated by a power law $d N / d I \propto I^{\delta}$ to a certain extent.

The left panels of Fig. 2 show that the profile of the intensity flux in the case of the Alfvén wave heated loop is very spiky and remains so when going from the top of the transition region towards the apex. This reflects the highly dynamical state of the corona, which is permeated by strong slow and fast shocks. Correspondingly, the intensity histograms on the left panels of Fig. 3 display power law indexes $\delta$ steeper than -2 , which remain roughly constant when shifting the observed region along the corona due to the uniformly distributed shock heating. Most of the heating thus comes from small energetic events (shocks). On the other hand, the intensity flux for the case of a loop with heating events concentrated at the footpoints is less spiky as shown by the middle panels of Fig. 2. The farther away we are looking from the top of the transition region, the less spiky the intensity profile becomes. The middle panels in Fig. 3 correspondingly show a power law index which close to the footpoint has a value of $\delta \sim \alpha=-1.86$ and decreases in magnitude to a value $\delta \sim-1.5$ at the apex. Both tendencies are due mainly to two reasons. Acoustic shocks dissipate rapidly leaving only weak shocks at the apex of the loop. The second reason is thermal conduction which, in the case of footpoint concentrated heating, is highest at the top of the transition region and damps the small temperature perturbations. This effect is more dramatic for uniform nanoflare heating, as shown by the right panels of Figs. 2 and 3. In this case maximum temperatures are found at the apex of the loop. Also, mean coronal temperatures are higher than for footpoint concentrated heating. Consequently, thermal conduction flattens intensity peaks much more effectively. The power law index of the intensity distribution rapidly becomes shallow and stays constant with a value close to -1 as the 'observed' region moves towards the apex, reflecting the low coronal dynamics.

The change in the power law index $\delta$ with respect to position along the loop for the case of footpoint concentrated heating events having a power law spectrum in energies implies that the measured heating events match roughly the heating events that are actually taking place in the loop only close to the transition region. Information of the small energetic events is lost in the high corona. The measurement of the power law index thus depends on the formation temperature of the observed emission line, making temperature a strong bias in the determination of this quantity. This could explain the existing discrepancies in measurements of the power law index when different emission lines are considered (cf. table 1 in Benz \& Krucker 2002; for more studies see e.g. Aschwanden 2004; Erdélyi \& Ballai 2007).

The power law index thus not only has information about the role of small heating events towards the overall heating but also about the location of the heating along the loop and about the operating heating mechanism. It is thus a powerful tool that can shed some light into the coronal heating problem.

Acknowledgements. P.A. would like to thank Drs T. Suzuki, H. Isobe, L. Heggland, T. Magara, R. Erdélyi, M. Carlsson, V. Hansteen for many fruitful discussions. This work was supported by the Grant-in-Aid for the 21st Century COE "Center for Diversity and Universality in Physics" from the Ministry of Education, Culture, Sports, Science and Technology (MEXT) of Japan, by a Grant from the International Astronomical Union, and by a Grant-in-Aid for Creative Scientific Research "The Basic Study of Space Weather Prediction" (17GS0208, Head Investigator: K. Shibata) from the Ministry of Education, Science, Sports, Technology, and Culture of Japan. The numerical calculations were carried out on Altix3700 BX2 at YITP in Kyoto University. 

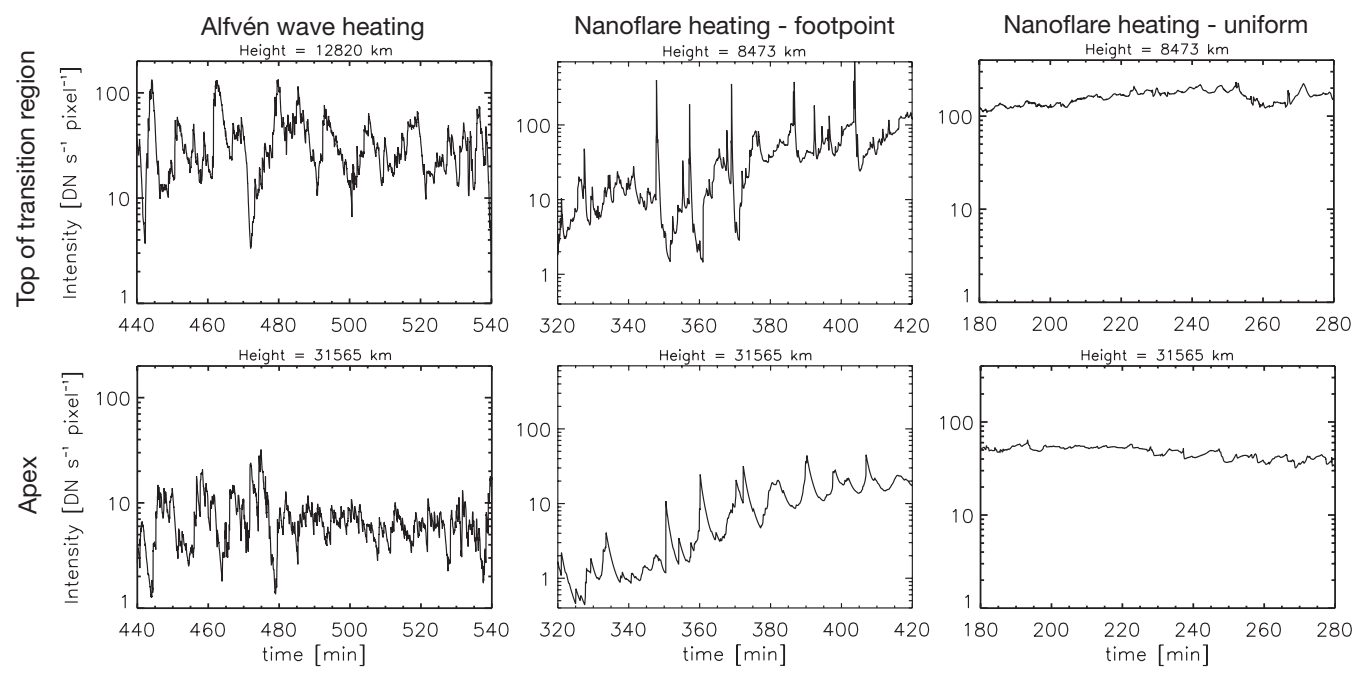

Figure 2. Intensity flux time series constructed from Hinode/XRT response function. Top and bottom row panels correspond, respectively, to the top of the transition region and apex of the loop as 'observed' regions (the corresponding height is indicated on the top of each panel). From left to right we have, respectively, a loop heated by Alfvén waves, a loop with heating events concentrated at the footpoints whose energies follow a power law distribution in frequency with an index of $\alpha=-1.8$, and a loop with heating events uniformly distributed along the loop and whose energies are uniformly distributed in frequency.
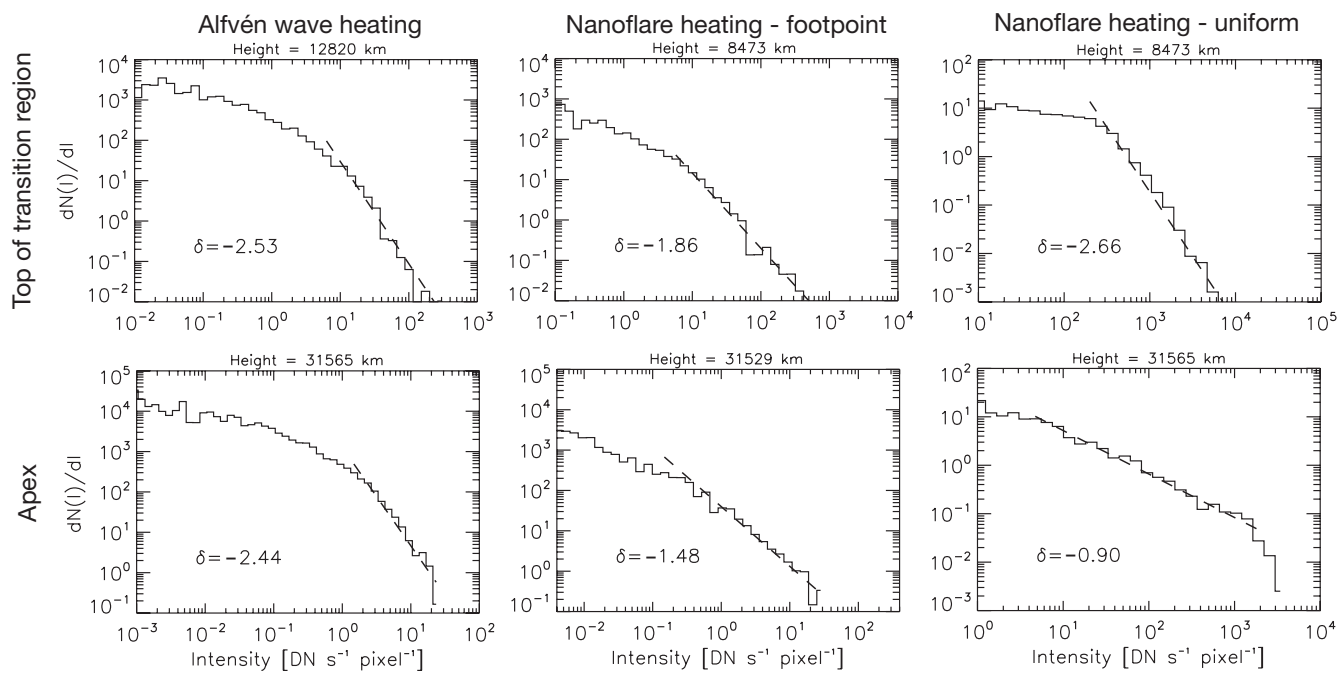

Figure 3. Intensity histograms constructed from the intensity flux time series in Fig. 2. Top and bottom row panels correspond, respectively, to the top of the transition region and apex of the loop as 'observed' regions (the corresponding height is indicated on the top of each panel). From left to right, column panels correspond, respectively, to a loop heated by Alfvén waves, a loop with heating events concentrated at the footpoints whose energies follow a power law distribution in frequency with an index of $\alpha=-1.8$, and a loop with heating events uniformly distributed along the loop and whose energies are uniformly distributed in frequency. 


\section{References}

Anderson, C.S., \& Athay, R.G. 1989, ApJ, 336, 1089

Aschwanden, M.J. 2001, ApJ (Letters), 559, L171

Aschwanden, M.J. 2004, Physics of the Solar Corona: An Introduction, Springer, Berlin

Alfvén, H. 1947, MNRAS, 107, 211

Benz, A.O., \& Krucker, S. 2002, ApJ, 568, 413

Erdélyi, R. 2004, A\&GG, 45, 34

Erdélyi, R., \& Ballai, I. 2007, AN, 328, 726

Evans, C.R., \& Hawley, J.F. 1988, ApJ, 332, 659

Hollweg, J.V., Jackson, S., \& Galloway, D. 1982, Solar Phys., 75, 35

Hori, K., Yokoyama, T., Kosugi, T., \& Shibata, K. 1997, ApJ, 489, 426

Hudson, H.S. 1991, Solar Phys., 133, 357

James, S. P., \& Erdélyi, R. 2002, A\&A, 393, L11

Katsukawa, Y., \& Tsuneta, S. 2001, ApJ, 557, 343

Krucker, S., \& Benz, A.O. 1998, ApJ (Letters), 501, L213

Kudoh, T., Matsumoto, R., \& Shibata, K. 1998, ApJ, 508, 186

Kudoh, T., \& Shibata, K. 1999, ApJ, 514, 493

Mendoza-Briceño, C.A., Erdélyi, R., \& Sigalotti, L.Di G. 2002, ApJ, 579, L49

Mendoza-Briceño, C.A., Sigalotti, L.Di G., \& Erdélyi, R. 2005, ApJ, 624, 1080

Mendoza-Briceño, C.A., \& Erdélyi, R. 2006, ApJ, 648, 722

Moriyasu, S., Kudoh, T., Yokoyama, T., \& Shibata, K. 2004, ApJ (Letters), 601, L107

Parker, E.N. 1988, ApJ, 330, 474

Parnell, C.E., \& Jupp, P.E. 2000, ApJ, 529, 554

Patsourakos, S., \& Vial J.-C. 2002, A\&A 385, 1073

Priest, E.R., Foley, C.R., Heyvaerts, J., Arber, T.D., Culhane J.L., \& Acton, L.W. 1998, Nature, 393,545

Reale, F. 2002, ApJ, 580, 566

Rosner R., Tucker, W.H., \& Vaiana, G.S. 1978, ApJ, 220, 643

Shibata, K., Tajima, T., Matsumoto, R., Horiuchi, T., Hanawa, T., Rosner, R., \& Uchida, Y. 1989a, ApJ, 338, 471

Shibata, K., Tajima, T., Steinolfson, R.S., \& Matsumoto, R. 1989b, ApJ, 345, 584

Shimizu, T. 1995, PASJ, 47, 251

Stone, J.M., \& Norman, M.L. 1992, ApJS, 80, 791

Takeuchi, A., \& Shibata, K. 2001, ApJ (Letters), 546, L73

Taroyan, Y., Bradshaw S.J., \& Doyle, J.G. 2006, A\& A, 446, 315

Taroyan, Y., Erdélyi, R., Doyle, J.G., \& Bradshaw, S.J. 2007, A\&A, 462, 331

Uchida, Y., \& Kaburaki, O. 1974, Solar Phys., 35, 451

Wentzel, D.G. 1974, Solar Phys., 39, 129

Yabe, T., \& Aoki, T. 1991, Comp. Phys. Comm., 66, 219 